\title{
El emprendimiento femenino: revisión de literatura
}

\section{Social Inclusion: A Fundamental Part Of Education for Integral Development}

\author{
Diana Alejandra Dussán ${ }^{1}$ \\ Derly Yurany Mosquera ${ }^{2}$ \\ Alexander Quintero ${ }^{3}$
}

\section{Resumen}

El propósito del presente artículo es analizar la producción científica en torno al emprendimiento femenino orientado hacia la actividad empresarial. Para tal cometido, se recurrió a las revisiones efectuadas por diversos autores al campo del conocimiento del emprendimiento. Igualmente, se examinó la producción intelectual generada en Colombia y la comunidad internacional en el periodo 2015-2017. Los resultados permiten ver que la participación de las mujeres empresarias es cada vez más importante en la transformación de las interacciones sociales. Así mismo, advierten que, mientras la mayoría de las mujeres crean empresa para lograr mayor independencia, la mayor parte de hombres lo hacen para mantener su nivel de ingresos. Una buena parte de los estudios sobre el emprendimiento se centran en hombres, incluso ya formados en la actividad empresarial, lo que deja un vacío para que el estudio actual se concentre en las mujeres empresarias.

Palabras claves: Emprendimiento, Emprendimiento femenino, Empresarismo, Empresariado femenino.

\section{Abstract}

The purpose of this article is to analyze the scientific production around female entrepreneurship oriented towards business activity. For such a task, reviews made by a variety of authors in the field of knowledge of entrepreneurship were used. Likewise, the intellectual production generated in Colombia and the international community in the period 2015-2017 was examined. The results evidence that the participation of women entrepreneurs is increasingly important in the transformation of social interactions. Likewise, these warn that, while most women create companies to achieve greater independence, most men do so to maintain their income level. Great part of the studies on entrepreneurship are focused on men, even men already trained in business activity, which leaves a gap for the current study to focus on women entrepreneurs.

Key words: Entrepreneurship, Female Entrepreneurship, Business, Female Business.

\footnotetext{
${ }^{1}$ Contadora pública y Magister en Administración, Universidad Surcolombiana. Correo electrónico diana.duba@hotmail.com

${ }^{2}$ Administradora de Empresas y Magister en Administración, Universidad Surcolombiana. Correo electrónico:

derlymosquerahuertas1940@gmail.com.

${ }^{3}$ Ph.d. en Administración, Magister en Historia Universidad Nacional, Magister en Organizaciones, Univalle, Especialista en Alta Gerencia. Profesor de tiempo completo Universidad Surcolombiana. Correo electrónico: alequin@usco.edu.co
} 


\section{Introducción}

Las mujeres empresarias están desempeñando un papel importante en la mayoría de los países desarrollados y también en algunos países en vía de desarrollo (Brush, 1992), aunque el número de mujeres empresarias sigue siendo relativamente pequeño en comparación con las empresas cuyos propietarios son hombres (Minniti \& Arenius, 2003) (Mutalib, Arshad, Ismail, \& Ahmad, 2015). Según Singh \& Raghuvanshi (2012) las mujeres siempre han contribuido a la productividad de las personas. No obstante, estudios del Banco Mundial dan cuenta que las mujeres reclaman solo el $10 \%$ de los ingresos generados por sus esfuerzos. Esta situación de desequilibrio refleja la transformación de las mujeres en una educación superior y agudiza sus habilidades en la gestión de negocios.

El número de mujeres empresarias sigue creciendo junto con la modernización. Una de las razones por las que las mujeres dejaron su carrera para convertirse en empresarias autónomas es porque no están satisfechas con su entorno laboral y no lograron conciliar la vida laboral y personal. La gestión de una empresa no es fácil para hombres y mujeres, pero las empresarias tienen que superar obstáculos más difíciles a lo largo del camino (Smith-Hunter, 2006). Por lo tanto, la fuerte motivación de las mujeres es el elemento más importante para mantener sus negocios y tener éxito.

Los beneficios del emprendimiento femenino son muchos y variados, y se han investigado con gran detalle en el pasado. Este artículo presenta una revisión del emprendimiento femenino en el mundo en desarrollo, como también en Colombia. El objetivo principal es discutir uno de los desarrollos económicos y sociales más significativos en el mundo, el auge del fenómeno de la mujer empresaria. El emprendimiento femenino debe ser estudiado como un campo separado por dos razones principales: Primero, la capacidad empresarial de la mujer es una importante fuente de crecimiento económico en la creación de nuevos puestos de trabajo y por ser genéticamente diferente, las mujeres aportan soluciones diferentes a las cuestiones de gestión y de negocios. Segundo, el emprendimiento femenino ha sido descuidado, en particular, en la investigación empresarial, aunque la igualdad de oportunidades entre hombres y mujeres en el ámbito empresarial no es una realidad a corto plazo, el progreso hacia su consecución podría verse facilitado por una mejor comprensión del impacto de la iniciativa empresarial femenina en la sociedad y en la economía.

Para un mejor entendimiento, en el presente artículo se aceptará la definición aportada por Farr-Warthon y Brunetto (2009): "las mujeres empresarias se definen como aquellas que usan sus conocimientos y recursos para desarrollar o crear nuevas oportunidades de negocios, que participan activamente en la gestión de sus negocios, y que poseen al menos el 50 por ciento del negocio y han estado en funcionamiento durante más de un año"

\section{Metodología}

La revisión se efectuó con base en la vigilancia tecnológica, de la cual existen diversos procedimientos validados por reconocidos autores (Berges-García, Meneses-Chaus y Martínez-Ortega, 2016; Giménez -Toledo y Román-Román, 2001). Identificación de herramientas: Para la elaboración del artículo se identificó y se accedió a la base de datos Scopus, la mayor base de datos de referencias y resúmenes revisados por pares principal que contiene información relacionada con el tema de investigación. Inicialmente se construyó un tesauro o lista de palabras con significados similares o sinónimos en español e inglés, que permitió establecer relaciones directas o indirectas con los conceptos a consultar, y a su vez construir con mayor precisión las ecuaciones de búsqueda: TITLE-ABS-KEY (emprendimiento and femenino) OR TITLE-ABS-KEY (female AND entrepreneurship) OR TITLE-ABS-KEY ('esprit AND d'entrepr) AND DOCTYPE (re) AND PUBYEAR > 2011 AND PUBYEAR < 2018 AND (LIMIT-TO (SUBJAREA, "BUSI") OR LIMIT-TO (SUBJAREA, "ECON"). 


\section{Resultados}

\section{Emprendimiento femenino}

El estudio académico del emprendimiento femenino comenzó a fines de la década de 1970, cuando las mujeres se unieron a la fuerza laboral profesional en mayor número. Chusmir, Moore, \& Adams (1990) informaron que la literatura de psicología proporcionó casi dos tercios de los estudios relacionados con cuestiones de género en las organizaciones. Carter, Anderson y Shaw (2001) concluyeron que muchos estudios fueron descriptivos y se centraron principalmente en las características demográficas, las motivaciones de las mujeres en los negocios y sus experiencias de propiedad empresarial. También señalaron que pocos estudios se basaron en trabajos previos para proporcionar conocimiento acumulativo de mujeres emprendedoras.

El estudio del emprendimiento femenino aún se encuentra en sus primeras etapas y numerosos investigadores han señalado la falta de una corriente de investigación sólida (Baker, Aldrich, \& Liou, (1997); Carter, Anderson, \& Shaw (2001); Terjesen, (2004). De 1976 a 2001, el 93\% de la investigación académica relacionada con el emprendimiento femenino que se encuentra en las revistas de emprendimiento fue de naturaleza empírica (Greene, Hart, Gatewood, Brush, \& Carter, 2003). Las revisiones de la literatura han sido mínimas, pero han proporcionado una gran comprensión del emprendimiento femenino. Moore (1990), quien publicó una de las primeras revisiones de literatura sobre el tema, llamó a los estudios que revisó "la primera ola de investigación seria "sobre el emprendimiento femenino". También informó que los datos disponibles en 1990 aún no eran suficientes para el desarrollo de la teoría. Baker, Aldrich y Liou (1997) criticaron a los medios de comunicación y las publicaciones académicas por no notar el tremendo crecimiento en el espíritu empresarial de las mujeres y concluyeron que "el espectacular progreso de las mujeres en la propiedad empresarial ha sido prácticamente invisible".
Probablemente la revisión más extensa de la literatura realizada por Greene et al., (2003) proporcionó información sobre los 25 años de estudio publicados en revistas de emprendimiento de 1976 a 2001. Un aspecto importante del estudio fue la inclusión y discusión de literatura basada en la teoría feminista, teoría social que aborda cuestiones de derechos políticos, económicos y sociales de las mujeres. La investigación feminista impulsada por la teoría ha proporcionado perspectivas adicionales, como el equilibrio entre el trabajo y la familia (Hoing-Haftel \& Martin, 1986), las diferencias de género (Chaganti, 1986) y los argumentos relacionados con sesgos metodológicos de investigaciones anteriores (Brush, 1992).

Parte de la literatura sobre el emprendimiento femenino se ha centrado únicamente en las emprendedoras, mientras que otras investigaciones han tratado de probar o refutar la noción de que existen diferencias significativas entre los emprendedores masculinos y femeninos con sus empresas. La evidencia de diferencias importantes y significativas es mínima, por ejemplo (Sabarwal \& Terrell, 2008) proporcionan evidencia de hallazgos contradictorios relacionados con las diferencias de género y atribuyen algunas diferencias a las clasificaciones de datos. Citan a (Chaganti \& Parasuraman, 1996), quienes concluyeron que los negocios propiedad de mujeres no tuvieron un desempeño inferior al de los hombres en términos de crecimiento laboral, mientras que otros concluyeron que sí (Bosma, van Praag, \& Thurik, 2004).

Fischer, Reuber, \& Dyke (1993) proporcionaron una visión general que sintetizó los elementos de sexo y género del estudio del emprendimiento. Los autores, que calificaron los hallazgos empíricos anteriores relacionados con el emprendimiento femenino como "diversos y a menudo contradictorios", concluyeron en su propio estudio sobre emprendedores masculinos y femeninos en las industrias manufactureras, de servicios y minoristas, que había pocas diferencias significativas en áreas como capacitación, motivación y experiencia. 
Varios investigadores atribuyen algunas de las lagunas y contradicciones en la literatura a la falta de investigación basada en la teoría sobre las mujeres empresarias (Moore, 1990; Brush, 1992; Baker, Aldrich y Liou, 1997). De Bruin, Brush y Welter (2006) informaron que los estudios sobre mujeres empresarias constituían sólo el 6-7\% de los estudios encontrados en las ocho principales revistas de iniciativa empresarial, atribuyeron la falta de una corriente de investigación sólida a razones como las percepciones de los investigadores y de la sociedad. Algunos investigadores han cuestionado la necesidad de una teoría separada sobre el emprendimiento femenino y piden conceptos teóricos que incorporen las experiencias distintivas de las mujeres (De Bruin, Brush \& Welter, 2006).

Para mejorar el flujo de investigación, Brush (1992) pidió un enfoque más holístico en el estudio de las mujeres emprendedoras, argumentando que la investigación que incorpora la perspectiva de que las propietarias de negocios consideran que sus negocios son cooperativos y las redes integradas de relaciones producirá mejores resultados. Por su parte, Muktar (2002) señaló que las diferencias entre los géneros están en términos de estilo de gestión, estructura organizativa de las empresas y el grado de empoderamiento en ellas.

Verhaul \& Thurik (2001), empleando un panel de 2000 personas, incluidas 500 mujeres que comenzaron nuevas empresas en los Países Bajos, descubrieron que las mujeres usaban poco capital inicial, aunque no había diferencia entre los tipos de capital.

Cowling \& Taylor (2001) encuentraron que las mujeres emprendedoras tienen una mejor educación que los hombres, y que en la década de 1990 los hombres ingresaron a la actividad empresarial en un número considerablemente mayor que las mujeres. En un estudio basado en 4.200 emprendedores, incluidas 405 mujeres, Du Rietz y Henrekson (2000) señalaron que la única área donde las mujeres tienen un rendimiento inferior, en comparación con los hombres, está en crecimiento en términos de ventas.
Por medio del estudio realizado por el Centro para la Investigación de Negocios de Mujeres (2009), el cual mide el impacto económico de los ocho millones de empresas que son de mujeres en los Estados Unidos, se logró establecer que el impacto económico estimado fue de $\$ 3$ billones anuales que se tradujo en la creación y/o mantenimiento de más de 23 millones de empleos, el $16 \%$ de todos los empleos fue en el mercado estadounidense. Este informe hace una declaración interesante que ilustra la importancia del emprendimiento femenino en la economía más fuerte del mundo: "Si las empresas estadounidenses pertenecientes a mujeres fueran su propio país, tendrían el quinto mayor PIB del mundo, muy por detrás de Alemania y por delante de los países incluyendo Francia, Reino Unido e Italia (Centro de Investigación Empresarial de la Mujer, 2009).

A pesar de esta tendencia, la "brecha de género" en esta actividad sigue siendo amplia. Las mujeres aún son menos propensas a ingresar a tal actividad que los hombres. Reynolds, Bygrave, Autio, Cox y Hay (2002) indican que el emprendimiento femenino varía significativamente entre los 37 países GEM 2002 (Global Entrepreneurship Monitor), que van desde $0.6 \%$ en Japón hasta el nivel más alto de $18.5 \%$ en Tailandia. Aunque en China, Sudáfrica y Tailandia la brecha es más estrecha que en otros países, concluyen que no hay país donde las mujeres sean más activas en el espíritu empresarial que los hombres. Kelly, Brush, Greene y Litovsky (2011) indican que la proporción de mujeres emprendedoras varía significativamente entre países, cifra oscila desde el $16 \%$ en la república China, hasta el $55 \%$ en Ghana, donde hay seis mujeres emprendedoras para un empresario masculino.

Minniti y Arenius (2003) afirman que la participación en actividades empresariales no varía entre países y géneros con respecto a la edad. Por ejemplo, entre hombres y mujeres, los años pico de participación en tales actividades son de 25 a 34 años. Los mayores de 55 años tienen la 
tasa de participación más baja, tanto entre hombres como mujeres.

\section{El impacto del emprendimiento femenino en la economía}

La motivación para considerar el emprendimiento femenino, tanto en los países en desarrollo como en los desarrollados, se deriva de la creciente comprensión de su importante papel en la creación, gestión y desarrollo de negocios como motor fundamental para el crecimiento económico (Acs, Bardasi, Estrin, \& Svejnar, 2011). El auge del emprendimiento femenino en las economías industrializadas es un fenómeno reciente. Aunque Estados Unidos es el país más cubierto con respecto al emprendimiento femenino, las emprendedoras en economías en transición como Hungría, Polonia, Rumania y Rusia, en América Latina, el sur de Asia y el sudeste asiático tienen un impacto significativo en las economías nacionales.

En la mayoría de las economías desarrolladas, los actores reales ya no son grandes empresas, sino pequeñas y medianas empresas que pueden proporcionar soluciones y responder a las necesidades derivadas de las nuevas tecnologías de la información. Aunque en el pasado la industrialización atraía a más mujeres a los negocios, los entornos actuales les ofrecen un nuevo papel para introducir la innovación y la creatividad, especialmente cuando las empresas se conectan con la calidad de vida. La modernización desafía las viejas regulaciones, barreras y viejos patrones de negocios que planteaban los principales obstáculos para el emprendimiento femenino.

En la era actual, que se caracteriza por una fuerte competencia nacional y mundial y un entorno económico inestable, las mujeres empresarias tienen un papel crucial en la creación de nuevos empleos y, por lo tanto, en la reducción del desempleo. Delmar y Holmquist (2004) afirman que las mujeres emprendedoras juegan un papel importante en la economía mundial e indican que en los Estados Unidos 6,4 millones de mujeres que trabajan por cuenta propia proporcionan trabajo a 9,2 millones de personas. Estos datos son impresionantes cuando se comparan con las empresas más grandes de Fortune 500 que juntas emplearon a 11.7 millones de personas (Woodroof, 1996).

En todo el mundo, el emprendimiento femenino contribuye a la estabilidad, al bienestar de las comunidades y brinda oportunidades económicas para grupos desfavorecidos, incluidas las mujeres, las personas con bajos salarios y las minorías. Otra contribución es la creación de riqueza y empleo.

Muchas mujeres en los países en desarrollo siguen siendo analfabetas y viven en comunidades pobres. Esta situación no les impide participar en las economías locales y convertirse en empresarias. Las pequeñas empresas les permiten mejorar sus vidas y las de muchos otros mediante la creación de nuevos empleos. La participación de las mujeres en la vida económica de los países en desarrollo contribuye a un entorno laboral más humano, cooperativo, equilibrado y agradable en las empresas dirigidas por mujeres, en el que está grabado el desarrollo individual. Hisrish y Brush (1988) sostienen que un número creciente de mujeres buscan penetrar en el mundo del emprendimiento, aunque esta actividad conlleva muchos riesgos y el gasto de tiempo y energía.

Numerosos estudios abordan la importancia del emprendimiento femenino en varias regiones del mundo. Kay, Gunterberg, Holz y Wolter (2003) argumentan que en Alemania más de un millón de mujeres son propietarias de empresas que proporcionan trabajo a dos millones de empleados. En el Reino Unido, las mujeres empresarias tienen una participación cada vez mayor en la población autónoma y una parte importante de la población de pequeñas empresas (Carter, Anderson y Shaw, 2001).

La OCDE (2003), en sus Estadísticas anuales de la fuerza laboral, indica los datos de las economías miembros con respecto a la distribución del género en el empleo. Según este informe, Turquía tiene la menor proporción de empleo femenino (13\%), mientras que Portugal tiene la mayor proporción 
(40\%). EE. UU. y Canadá ocupan el puesto más alto, con una tasa de 38 a 40\%. Los países escandinavos tienen una participación del 20-25\%. Chun (1999) indica que las mujeres empleadoras en los países de APEC (Foro de Cooperación Económica Asia-Pacífico) tienen, en promedio, una participación del $30 \%$ en el mercado laboral.

Saavedra (2001) proporciona estadísticas sobre mujeres autónomas en Argentina, Brasil y Costa Rica que indican que el trabajo por cuenta propia representa una opción real e importante para ellas, aunque Brasil lidera a Argentina y Costa Rica. Hoy en día, el autoempleo está aumentando en Brasil y Costa Rica. El informe de 2012 de la Asociación de Estados del Caribe (AEC) indica que en América Latina las mujeres tienen más probabilidades de ser emprendedoras que en otras regiones, y que la división allí entre hombres y mujeres empresarios es del $24 \%$ frente al $43 \%$ en Asia y el $45 \%$ en Europa.

Con base en estos datos, se puede concluir que el emprendimiento femenino tiene un fuerte efecto en las economías al aumentar el empleo $\mathrm{y}$ las ventas. Las mujeres emprendedoras juegan un papel importante en el desarrollo y el crecimiento económico. No obstante, existe dificultad para estimar el impacto real del fenómeno discutido en la economía. Delmar y Holmquist (2004) sostienen que existe una falta de conocimiento sobre el impacto real del emprendimiento femenino en el crecimiento de la economía en la mayoría de los países miembros de la OCDE.

\section{El emprendimiento en Colombia}

De acuerdo con el Informe del GEM 2015, en Colombia los emprendimientos por necesidad en las mujeres son del $34,5 \%$ y el $64,6 \%$ lo realiza por oportunidad; por el contrario, la tasa de emprendimiento por necesidad en los varones es del $28 \%$ y por oportunidad el $71,5 \%$.

Por otro lado, en Colombia la participación de la mujer en el mercado laboral se ha venido incrementando. Según el Departamento Nacional de Planeación, en 1978 la tasa de participación de los hombres alcanzó el 74,6\% y para las mujeres el 25,4\%. Para el año 2017 esta última alcanzó un $54,5 \%$ según el DANE.

En Colombia para fomentar la cultura de emprendimiento se expidió la ley 1014 del 2006 con la cual se generaron condiciones para el surgimiento de fondos de capital semilla, fondos de capital de riesgo y ángeles inversores para apoyar a las nuevas empresas. Colombia ha sido catalogado como un país con un alto comportamiento emprendedor. Para el caso femenino, según estudio del GEM para el 2012, la Tasa de Actividad Emprendedora (TEA) para hombres fue de $26,9 \%$, mientras que para mujeres fue de 16,3\%. Para el año 2016 ésta alcanzó el $25 \%$. Esta es una característica de los países con PIB per cápita bajo en donde el desempleo conlleva al ingreso en actividades empresariales por autoempleo o por necesidad.

Varela (2008) considera que las mujeres en Colombia ven oportunidades para emprender y crecer e inician el emprendimiento motivadas en generar ingresos y aprovechar su tiempo libre mientras que para los hombres es ganar independencia financiera. Las primeras son más sensibles al riesgo y buscan mercados potencialmente estables, por el contrario, los hombres son más dados al riesgo y prefieren mayores ganancias. Solo el $30,2 \%$ de las mujeres aceptan trabajar en actividades de alto riesgo. Según el estudio del GEM 2014, la mayoría de las mujeres afirman que crean empresa para lograr mayor independencia mientras que la mayoría de los hombres dicen que es para mantener su nivel de ingresos. Por lo cual son necesarios programas que estimulen el emprendimiento femenino en el país al igual que políticas de equidad de género.

\section{Conclusiones}

No hay duda de que el emprendimiento femenino es uno de los fenómenos sobresalientes en la economía moderna. Un número creciente de mujeres que participan en actividades empresariales refleja la transformación en los ámbitos económico y social. Las mujeres están cruzando cada vez más las barreras económicas y 
sociales. Tienen mayor confianza en sus habilidades y destrezas para construir sus empresas por igual, si no con más éxito, que los hombres.

La motivación individual, la estructura familiar, la educación, la demografía, el desempleo y los entornos sociales y económicos son los principales factores que influyen en el emprendimiento femenino. Estos factores son los principales determinantes de las diferencias entre mujeres y hombres con respecto a las percepciones de riesgo en el proceso empresarial.

Las mujeres emprendedoras enfrentan varios obstáculos a lo largo del ciclo de vida de sus negocios: la ausencia de posibilidades de evaluación comparativa, la falta de capital financiero y social, de experiencia, tiempo, discriminación de género, actitudes de estereotipo y, finalmente, problemas domésticos.

Muchos formuladores de políticas perciben el emprendimiento femenino como un fenómeno que contribuye al crecimiento económico. Esta percepción debe ir acompañada de especial atención, apoyo y tratamiento. En realidad, hay mucho más por lograr para alentar y ayudar a las mujeres que aspiran a embarcarse en una actividad empresarial.

A pesar del rápido crecimiento de las mujeres en trabajos profesionales y gerenciales, la brecha de género en el espíritu empresarial sigue siendo significativa. En promedio, los hombres tienen un 50 por ciento más de probabilidades que las mujeres de convertirse en empresarios.

En los países de bajos ingresos, el crecimiento de la población y el analfabetismo femenino se asocian con mayores niveles de actividad empresarial tanto para hombres como para mujeres. Por el contrario, la seguridad económica, en forma de pagos de asistencia social o empleo estable, parece desalentar el espíritu empresarial entre los géneros en países de ingresos altos y bajos.

En comparación con los hombres, las mujeres empresarias usan cantidades más pequeñas de capital inicial, una proporción más pequeña de acciones y más préstamos bancarios. Además, las empresas propiedad de mujeres tienden a ser más pequeñas y a crecer más lentamente que las de los hombres, lo que sugiere diferencias de género en el valor asociado a la expansión.

Finalmente, la distribución del empleo femenino entre los sectores y la participación de las mujeres en puestos directivos también se correlacionan con el nivel de emprendimiento femenino, aunque sus efectos difieren. En los países de altos ingresos, el porcentaje de mujeres en puestos directivos públicos y privados se correlaciona positivamente con la oportunidad de emprender, mientras que en los países de bajos ingresos la correlación es negativa.

\section{Referencias}

Acs, Z. J., Bardasi, E., Estrin, S., \& Svejnar, J. (2011). Introduction to special issue of Small Business Economics on female entrepreneurship in developed and developing economies. Small Business Economics, 37(4), 393-396.

Baker, T., E. aldrich, H., \& Nina, L. (1997). Invisible entrepreneurs: The neglect of women business owners by mass media and scholarly journals in the USA. Entrepreneurship \& Regional Development, 9(3), 221-238.

Baker, T., E. aldrich, H., \& Nina, L. (1997). Invisible entrepreneurs: The neglect of women business owners by mass media and scholarly journals in the USA. Entrepreneurship \& Regional Development, 9(3), 221-238.

Bosma, N., Van Praag, M., Thurik, R., \& De Wit, G. (2004). The value of human and social capital investments for the business performance of startups. Small Business Economics, 23(3), 227-236.

Brush, C. G. (1992). Research on women business owners: Past trends, a new perspective and future directions. Entrepreneurship theory and practice, 16(4), 5-30. 
Buskirk, B. \& Lavik, M. (2004). Entrepreneurial marketing: real stories and survival strategies.

Carter, S. L., Anderson, S., \& Shaw, E. (2001). Women's business ownership: A review of the academic, popular and internet literature.

The Economic Impact of Women-Owned Businesses in the United States https://www.nwbc.gov/2009/10/27/the-economi c-impact-of-women-owned-businesses-in-the-unite d-states/

Chaganti, R. (1986). Management in women-owned enterprises. Journal of Small Business Management.

Chaganti, R., \& Parasuraman, S. (1996). Un estudio de los impactos de género en el desempeño empresarial y en los patrones de gestión de las pequeñas empresas. ntrepreneurship, Theory \& Practice, Winter.

Chusmir, L., Moore, D., \& Adams, J. (1990). Investigación sobre mujeres trabajadoras: una boleta de calificaciones de 22 revistas. Roles sexuales: un diario de investigación, 167-175.

Drucker, P. (1985). nnovación y emprendedurismo; Práctica y principios.

Dzisi, S. (2008). Actividades empresariales de mujeres indígenas africanas: un caso de Ghana. Revista de comunidades emprendedoras: personas y lugares en la economía global, 254-264.

Farr-Wharton, R., \& Brunetto, Y. (2009). Mujeres emprendedoras como gerentes: el papel del capital social en la facilitación de una cultura de aprendizaje. Género en la gestión: una revista internacional, 14-31.

Fischer, E., Reuber, A., \& Dyke, L. (1993). Una descripción teórica y extensión de la investigación sobre sexo, género y emprendimiento. Journal of Business Venturing.

Greene, P., Hart, M., Gatewood, E., Brush, C., \& Carter, N. (2003). College of Charleston.

Obtenido de Women Entrepreneurs: Moving Front and Center: An Overview of Research and Theory.: http://www.cofc.edu/entrepreneurconsortium/ resources/research_women.php

Herbert, R., \& Link, A. (1988). The Entrepreneur - Mainstream Views and Radical Critiques. New York: Praeger.

Hoing-Haftel, \& Martin. (1986). ¿Está la mujer empresaria en desventaja? Thrust.

Kirzner, I. (1997). Descubrimiento de emprendimiento y el proceso de mercado competitivo: un enfoque austriaco. Revista de Literatura Económica, 60-85.

Leibenstein, H. (1968). Emprendimiento y desarrollo. . Revista de Economia Americana.

Minniti, M., \& Arenius, P. (2003). Mujeres emprendedoras. La ventaja empresarial de las naciones. Primer simposio mundial anual sobre iniciativa empresarial.

Moore, D. (1990). Un examen de la investigación actual sobre la empresaria femenina: estrategias de investigación sugeridas para la década de 1990. Revista de ética empresarial, 275-281.

Mutalib, R., Arshad, R., Ismail, N., \& Ahmad, Z. (2015). La mujer y el espíritu emprendedor: Panorama general de los programas de empresariado femenino en Malasia.

Pickle, H., \& Abrahamson, R. (1990). Gestión de pequeñas empresas.

Sabarwal, S., \& Terrell, K. (2008). ¿Importa el género en el desempeño de la empresa? Evidencia de Europa del Este y Asia Central, Documento de debate. Instituto de Estudios Laborales (IZA).

Schumpeter, J. (1942). Capitalismo, socialismo y democracia.

Singh, G., \& Belwal, R. (2008). Emprendimiento y pymes en Etiopía: evaluación del papel, las 
perspectivas y los problemas que enfrentan las mujeres en este sector emergente. Género en la gestión: Revista Internacional, 120-136.

Singh, R., \& Raghuvanshi, N. (2012). Problemas de emprendimiento de mujeres, desafíos y empoderamiento a través de grupos de autoayuda. Revista Internacional de Estudios Democráticos y de Desarrollo, 45-58.

Smith-Hunter, A. (2006). Mujeres emprendedoras a través de líneas raciales: cuestiones de capital humano, capital financiero y estructuras de red. Revista Edward Elgar.

Terjesen, S. (2004). Mujeres propietarias de empresas: Una revisión de la última década de investigación. Academia de AdministraciónDivisión de Empresas.

Walker, D., \& Joyner, B. (1999). El emprendimiento femenino y el proceso de mercado: consideraciones de políticas públicas basadas en el género. Revista de emprendimiento del desarrollo, 95-116. 